\title{
Wargaming Future National Security Threats Posed by Emerg- ing Vector-Borne Diseases
}

\author{
Deon Canyon ${ }^{1}$
}

1 Daniel K. Inouye Asia-Pacific Center for Security Studies, Honolulu, Hawaii; canyond@apcss.org

\begin{abstract}
The emergence of existing and novel vector-borne disease pathogens is highly unpredictable because there are so many possible causal factors of sociological, ecological, biological, behavioral and political origin. Traditional data-driven forecasting tools have limited use in circumstances featuring low-probability crises for which trend data is unreliable and non-predictive. Commercial and security sectors have been quick to adopt various forms of wargames to address this gap and provide intelligent insights on possible outcomes in the short, mid and long term future. The lack of commitment and action against vector-borne diseases by governments and local authorities requires a new approach that presents risk in terms of potential actions, possible outcomes and resulting consequences.
\end{abstract}

Keywords: strategy; national security; competitive gaming; wargaming; military; defense force; arboviruses; crisis management

\section{Introduction}

The year 2020 was notable for the SARS-CoV-2 pandemic with 83,927,835 reported cases and 1,834,358 deaths, but few people beyond infectious disease experts were aware that in the same year, hundreds of millions were afflicted by vector-borne diseases, such as malaria and dengue, that killed over 700,000 people [1].

Vector-borne diseases (VBD) have been a threat to human health for centuries and while many people consider diseases like malaria to be an African problem, arboviruses such as West Nile virus, Dengue fever, Zika virus and Chikungunya virus have emerged and spread in North America. Virtually all arboviruses have their origins in wildlife transmission cycles and human infection occurs in three ways. Firstly, a bridging vector, living between wildlife and humans, may become infected by the wildlife cycle and bring a pathogen over to the human side (e.g. Yellow fever). Second, a virus may amplify in domestic animal reservoirs to the extent that humans living nearby become incidentally infected (e.g. Japanese encephalitis). In this situation, humans are an end-point for the pathogen and do not contribute to further transmission. Thirdly, a pathogen may move from a wildlife cycle to a distinct human cycle involving anthropophilic vectors that live in urban settings (e.g. dengue). This category generates the most human disease and is a critical focus for public health interventions.

\section{Predictability of vector-borne diseases in the US}

Pathogen emergence is highly unpredictable because there are many possible causal factors. Humans have become more susceptible to infection through these three possible transmission pathways for two main reasons. Changing ecological contexts due to globalization, climate change and human modification of ecosystems have resulted in shifting habitats, exposure to new vectors, and the movement of vectors around the world into new habitats facilitated by globalization. Changing social contexts due to evolving population demographics and increasing population movement have increased exposure to new vectors and new wildlife cycles, as well as made it easier for pathogens to spread around the world in human hosts. Long-term behavioral changes in human movement 
and aggregation due to the COVID-19 pandemic may have a further impact on the way that arboviruses transmit.

For instance, West Nile virus (WNV) did not exist in the United before 1999, and nobody predicted that it would become the most common vector-borne disease in the US [2]. The presence of culicine mosquito vectors and many species of avian vertebrate hosts enabled the virus to spread across the country in five years [3]. From its introduction into the US in 1999 to 2017, there have been 7,000,000 infections with 22,399 cases of neuroinvasive disease and 2,034 deaths. Although WNV outbreaks often occur during heat waves, they are otherwise unpredictable $[4,5]$. Communities have often fail to implement adequate surveillance, and neglect mosquito control due to a lack of skilled personnel, funding and concerns about pesticide use [6].

Dengue fever (DF) is the most common arbovirus disease worldwide and has posed an important public health threat in the US since 2009 due largely to several thousand dengue-infected tourists and travelers entering the US annually [7]. During 2010-2017, totals of 5,009 travel-associated and 378 locally acquired confirmed or probable dengue cases and 3 deaths were reported to ArboNET [8]. The preferred habitat of its primary vector, Aedes aegypti, generally lies between the $10^{\circ}$ isotherms above and below the equator, however, it has been known to emerge, totally unexpected, in temperate states such as New York. Further, dengue is growing in endemic areas, such as Puerto Rico and the US Virgin Islands [3].

Chikungunya virus made its first surprising appearance when it was locally transmitted in the Americas in the Caribbean in 2013 [9]. Spreading via the same vector as Dengue, this pathogen caused over a million infections in throughout Latin America and 2,400 cases in the US within a year [10]. Chikungunya virus is now a health concern in US where mosquito vectors exist and autochthonous transmission regularly occurs in Puerto Rico [11].

Zika virus is spread by the same vectors that transmit Dengue and Chikungunya, but it sparked great concern when it was found to cause infection by passing from a pregnant woman to her fetus, passing between sexual partners, and rarely passing between nonsexual partners. The common symptoms akin to mild Dengue were expected but the world had never seen an arbovirus that caused fetal infection resulting in brain abnormalities, such as microcephaly.

\section{Traditional forecasting fails when faced with complex, unpredictable crises}

As we face these highly unpredictable occurrences of pathogen emergence and effects, we have almost no new vector control methods, very few cures or vaccines for arboviruses and other vector-borne pathogens, drug resistance is growing, insecticide resistance is growing, and scientists with the necessary skills to do these things are decreasing day by day. When WNV emerged in the US, arboviral disease surveillance systems received a boost in support. However, when WNV infection became a "new normal," attention to and investment in surveillance, research, and prevention all deteriorated and the gap between capacity and ability to respond has grown ever since [12]. Fortunately, large outbreaks of Dengue, Chikungunya and Zika have not occurred in the past few years, but this only leads to diminished resources, complacency and the loss of workplace skills and trained personnel.

Vector-borne diseases disrupt health security as they are an important episodic cause of death, disease burden and health inequity. They hamper socioeconomic development and place a strain on health services. Controlling these diseases and gaining insight into how they might behave in the future is vital to health security. However, the constantly evolving, volatile, complex and changing world often presents challenging impacts to the crisis management community.

The use of mathematical models, the mainstay of forecasting endeavors, is only possible in environments in which curve balls and black swans don't exist, and where prob- 
ability of occurrence is high, when outcomes are well-known, and future trends are dependable. Crisis scholars don't use them because none of these conditions exist for real crises. Mathematical forecasting fails when major crises are typically one off events for which there are little data. Every pandemic is different and every hurricane is accompanied by a unique set of challenges. The solutions to these crises do not exist on a shelf of best-practices. If no commitment has been made to proactively thinking about possible crises, all responses are completely reactive trial and error affairs. Gaining insights into possible future vector-borne events requires not only an understanding of complex biological and ecological interactions, but creative and collaborative systems thinking. It requires a commitment to new thinking.

\section{Providing valuable future insights to decision-makers}

As our crises grow in frequency, intensity and complexity, decision-makers are faced with rapidly accumulating piles of data that quickly diminish in reliability and usefulness as a crisis progresses. The challenge for them is how to gain insight into what crises are coming in the short term future ( 1 to 5 years) when a decision needs to be made now to prepare for them. The question is, when traditional approaches fail, how can we generate sufficient competitive intelligence to make intelligent decisions that address short-term threats?

The need to forecast in uncertain situations and the failure of traditional forecasting tools have driven the commercial sector towards Business Wargaming and the defense sector towards Competitive Security Gaming [13.14]. These are quite different from strategic foresight, which is a common methodology for gaining insights beyond 5 years [15]. Competitive Security Games reframe wargaming in terms of strategic, operational and tactical competition rather than conflict, and their indicators of success relate closely to national security. They can just as easily be used to address the threat imposed by emerging VBDs.

The games for evaluating complex threats have themselves emerged from various sectors as a consequence of the type of challenges they face. Some migrate from the military into business and politics while most originate in the highly competitive business environment. Succeeding in these games requires systematic, multiple-source data collection, appraisal to collate valid information, fusion to create actionable intelligence, and making sure the decision makers are informed. In the absence of sound quantitative models, the intent is to develop 'strategic choices driven by insights rather than by gut feeling, conventional wisdom, or the loudest voice in the room [16].'

In wargames, natural disasters and pathogens can be injected by game controllers, or they can be played by a person or a team. The former construct typically promotes the implementation of standard, familiar responses, but the latter can result in constant pressure applied by out-of-box, emergent thinking. Providing that the time setting is set for a more strategic game, this pressure drives novel thinking by forcing people to face threats they have never faced, which provides them with the opportunity to devise approaches they have never considered. They game environment is not only safe, it is cheap and all imaginable approaches can be trialed and considered.

In the context vector-borne diseases, a Competitive Security Game would test the behavior and outcomes (strategy) presented by a novel emerging VBD by exposing it to three distinct lines of force (representing different interest groups - e.g. medical, governance, scientific). These forces operate independently and in concert as they challenge the VBD strategy in an environment where various uncontrollable factors may arise (due to player actions, action consequences, and control injects) to render the situation more complex, urgent and risky. These three forces must be limited to entities that have a direct bearing on the strategy under review.

In addition to control and assessment cells, there are typically four active player cells. One represents the VBD that is "testing its strategy" (Strategist Cell), and three represent competing nations or entities that challenge that strategy (Competitor Cells). Other sub- 
strategist or sub-competitor cells may be involved if they are essential to testing the strategy under investigation. Figure 1 shows the game flow of a VBD Competitive Security Game.

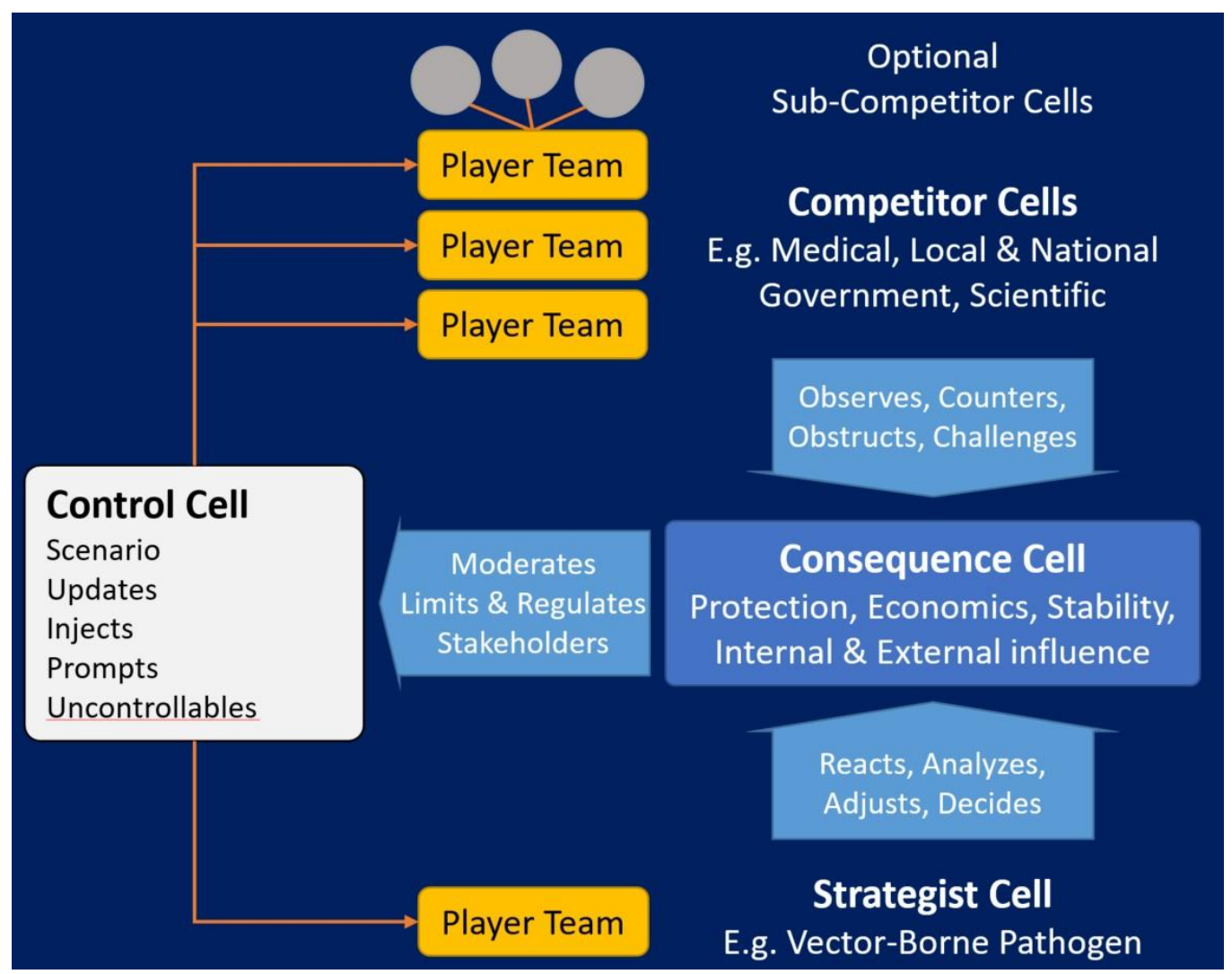

Figure 1. Cell roles and flow of a Competitive Security Game as it runs in practice [17].

Success can be evaluated by determining the impact of player actions on the VBD in terms of: Protection: Level of threat to national population; 2) Economics: Cost/Level of resources required/available; 3) Stability: in terms of population compliance with medical and scientific advice; 4) Internal influence: in terms of public opinion; and 5) External influence: in terms of the impact of the VBD and national actions on alliances, partnerships, and international reputation.

\section{Benefits of competitive gaming for vector-borne disease forecasting}

Competitive Security Gaming reframes the struggle to manage disease pathogens in terms of strategic, operational and tactical competition rather than man versus non-thinking pathogen. Like business wargames, they can generate high-end, qualitative analytics that ensure quality outcomes and insights. But unlike business games, they focus on higher-level strategy and are not solely focused on financial gain. They also increase the chance of success in many other ways by: challenging assumptions; revealing action consequences; allowing the exploration of alternative strategies; stimulating innovative thinking; uniting ideas, people and plans; improve interagency coordination; identifying blind spots; promoting resilience; raising issue awareness; team building; and spotlighting talent.

One of the greatest assets of competitive security games is their ability to reveal the strengths and weaknesses of the entity sitting in the Strategy Cell. Fresh thinking on potential pathogen capacities, mechanisms, and blind spots is the highest form of intelli- 
gence analysis [18]. Information on faulty assumptions is an added bonus that is particularly helpful when it comes to modifications in VBD management strategies that can correct internal deficiencies and neutralize external threats.

\section{Usefulness of this information to the Defense sector}

The evolving socio-ecological environment is likely to require ministries of defense to assess potential impacts of future vector-borne diseases on assumptions, doctrine and operational constructs [19]. In times of peace and war, nations gain goodwill from crisis assistance operations and militaries get the opportunity to test various systems and joint service interoperability. Many of these operations take place in disease endemic areas that have consequences for force protection and level of commitment.

The coordination of international military training exercises will become increasingly difficult as they deal with VBD changes due to climate change and increasing global population movement. Military VBD units will become more integrated with planners as the demand for VBD skills and training increases. Health protection considerations in these training program will increase. Surges in VBDs will alter the parameters and requirements for exercises, wargames and training for multiple, simultaneous crises.

More Defense personnel will be operating in VBD-degraded environments. The threat of VBDs and their actual spread will require medical care, prophylactics, personal protective equipment and support for psychological well-being.

Climate change may result in some Defense installations being located in environments that become more attractive to and supportive of disease vectors. Infrastructure and vehicles may require modification to reduce exposure to disease vectors thus increasing energy footprints and environmental impact. Degradation of infrastructure may also pose a risk if it provides more vector breeding sites.

Ensuring public buy-in is fundamental when disease vectors rely on public behaviors for survival and dissemination. Creating social narratives that enlist public support has always been important in changing people's behavior and getting them to advocate for more governmental resources and commitment to action. Internationally, nations seen to be taking proactive steps to minimizing the threatening outcomes of a changing climate will be able to project more diplomatic influence.

The shrinking number of scientists capable of preparing for episodic VBD outbreaks could be braced by a corresponding increase in personnel, roles and activities for the military. The potential for VBDs to become national crises speaks to the need for a larger skilled civilian force, however, the episodic nature of the threat renders it more suitable for the military, who constantly operate internationally in endemic areas exposed to the same threats.

These endeavors combined with the transboundary threat posed by VBDs requires joint-force action, inter-agency collaboration, and international interoperability. Humanitarian assistance and disaster relief exercises and operations have been a mainstay for practicing interoperability, but as nations become more self-sufficient, pathogen response may grow in importance.

Precautions against VBDs may even be useful when it comes to reducing vulnerability to miniature airborne drones.

Ultimately, any VBD wargaming effort should aim to establish the existing level of government knowledge, characterize the context, drivers and goals of VBD policy, identify and prioritize challenges and opportunities, and suggest policy actions to resolve the issues and their consequences.

Vector-borne diseases continue to evolve and to disrupt nations around the globe and they are being exacerbated by climate change and changing socio-ecological behaviors and conditions. Resilience depends on our ability to gain insight into how the various driving forces will play out in the years to come. In facing this challenge, governments must develop more insightful and coherent policies that include a role for security sectors. 
Considering what is coming in the future must become integral to our management practices so that we can demonstrate thought leadership as we strive to mitigate and prepare for vector-borne black swans [20].

Funding: “This research received no external funding"

Institutional Review Board Statement: "Not applicable"

Informed Consent Statement: “Not applicable"

Data Availability Statement: "Not applicable"

Acknowledgments: "Not applicable"

Conflicts of Interest: "Not applicable"

\section{References}

1. WHO. https://www.who.int/news-room/fact-sheets/detail/vector-borne-diseases (accessed on 16/03/2021).

2. Curren EJ, Lehman J, Kolsin J, Walker WL, Martin SW, Staples JE, Hills SL, Gould SV, Rabe IB, Fischer M, et al. West Nile virus and other nationally notifiable arboviral diseases - United States, 2017. MMWR. Morb. Mortal. Wkly. Rep. 2018;67:1137-1142.

3. Petersen LR, Hayes EB. West Nile virus in the Americas. Medical Clinics of North America 2008;92(6):1307-1322.

4. Kilpatrick AM, Meola MA, Moudy RM, Kramer LD. Temperature, viral genetics, and the transmission of West Nile virus by Culex pipiens mosquitoes. PLoS Pathogens 2008;4(6):e1000092.

5. Healy JM, Reisen WK, Kramer VL, Fischer M, Lindsey NP, Nasci RS, Macedo PA, White G, Takahashi R, Khang L, Barker CM. Comparison of the efficiency and cost of West Nile virus surveillance methods in California. Vector Borne Zoonotic Diseases 2015;15(2):147-155.

6. Chung WM, Buseman CM, Joyner SN, Hughes SM, Fomby TB, Luby JP, Haley RW. The 2012 West Nile encephalitis epidemic in Dallas, Texas. Journal of the American Medical Association 2013;310(3):297-307.

7. Mohammed HP, Ramos MM, Rivera A, Johansson M, Munoz-Jordan JL, Sun W, Tomashek KM. Travel-associated dengue infections in the United States, 1996 to 2005. Journal of Travel Medicine 2010;17(1):8-14.

8. Rivera A, Adams LE, Sharp TM, Lehman JA, Waterman SH, Paz-Bailey G. Travel-Associated and Locally Acquired Dengue Cases - United States, 2010-2017. MMWR Morb Mortal Wkly Rep 2020;69:149-154.

9. Leparc-Goffart I, Nougairede A, Cassadou S, Prat C, de Lamballerie X. Chikungunya in the Americas. Lancet 2014;383(9916):514.

10. Sharp TM, Roth NM, Torres J, Ryff KR, Perez Rodriguez NM, Mercado C, Pilar Diaz Padro MD, Ramos M, Phillips R, Lozier $\mathrm{M}$, et al. Chikungunya cases identified through passive surveillance and household investigations - Puerto Rico, May 5-August 12, 2014. Morbidity and Mortality Weekly Report 2014;63(48):1121-1128.

11. Centers for Disease Control and Prevention. https://www.cdc.gov/chikungunya/geo/united-states.html (accessed on 16/03/2021).

12. Petersen LR, Nasci RS, Beard CB, Massung RF. A9 - Emerging Vector-Borne Diseases in the United States: What Is Next, and Are We Prepared?" National Academies of Sciences, Engineering, and Medicine. 2016. Global Health Impacts of Vector-Borne Diseases: Workshop Summary. Washington, DC: The National Academies Press. doi: 10.17226/21792.

13. Kurtz J. Business wargaming: simulations guide crucial strategy decisions. Strategy \& Leadership 2003;31(6):12-21.

14. Canyon D. Strategic competition, national security and the need for 'competitive intelligence'. Security Nexus 2020;21:14 Oct.

15. Canyon D. Simplifying complexity with strategic foresight and scenario planning. Security Nexus 2021;22:7 Jan.

16. SCIP. https://www.scip.org/page/About-Us (accessed on 16/03/2021).

17. Canyon D. Competitive security gaming: rethinking wargaming to provide competitive intelligence that informs strategic competition and national security. Security Nexus 2020;21:24 Nov.

18. Gilad B. Business War Games. The Career Press: Pompton Plains NJ, 2008.

19. Cox K, Knack A, Robson M, Adger N, Paille P, Freeman J, Black J, Harris R. A changing climate: exploring implications of climate change for UK defence and security. RAND Europe.

20. Canyon D. The value of thought leadership in a world in crisis. Security Nexus 2020;21:3 Apr. 